\title{
Hepatic Steatosis Is Associated with Elevated Serum Iron in Patients with Obesity and Improves after Laparoscopic Sleeve Gastrectomy
}

\author{
Bingwei Ma ${ }^{a}$ c Hang Sun ${ }^{a}$ Bing Zhu ${ }^{a}$ Shilin Wang ${ }^{d}$ Lei Du ${ }^{a}$ \\ Xingchun Wang ${ }^{a, b}$ Shen $\mathrm{Qu}^{\mathrm{a}, \mathrm{b}}$
}

${ }^{a}$ Department of Endocrinology and Metabolism, Tongji University School of Medicine, Shanghai Tenth People's Hospital Affiliated to Tongji University, Shanghai, China; ${ }^{\text {T} T h y r o i d ~ R e s e a r c h ~ C e n t e r ~ o f ~ S h a n g h a i, ~ S h a n g h a i, ~ C h i n a ; ~}$ 'Department of Gastrointestinal Surgery, Tongji University School of Medicine, Shanghai Tenth People's Hospital Affiliated to Tongji University, Shanghai, China; ${ }^{d}$ Department of Liver and Gallbladder Surgery, Tongji University School of Medicine, Shanghai Tenth People's Hospital Affiliated to Tongji University, Shanghai, China

\section{Keywords}

Iron · Hepatic fat deposition - Laparoscopic sleeve

gastrectomy · Obesity

\begin{abstract}
Background: Iron is closely related to metabolism. However, the relationship between iron and hepatic steatosis has not been fully elucidated. Objective: We aimed to investigate the triangular relationship between iron and hepatic steatosis and laparoscopic sleeve gastrectomy (LSG) in patients with obesity. Methods: A total of 297 patients with obesity and 43 healthy individuals with a normal BMI were enrolled. Eighty-two patients underwent LSG. Anthropometrics, glucose-lipid metabolic markers, and hepatic steatosis assessed by FibroScan (CAP value and E value) were measured at baseline, and again at follow-up time intervals of 6 months and 1 year after surgery. Results: (1) Iron was significantly higher in patients with obesity or overweight than in the individuals with normal BMI $(8.18 \pm 1.47$ vs. $7.46 \pm 0.99 \mathrm{mmol} / \mathrm{L}$, $p=0.002$ ). Iron was also higher in subjects with high blood pressure, dyslipidemia, and hyperuricemia than non-corresponding disorders (all $p<0.05$ ). Moreover, iron was significantly higher in the severe than mild or moderate non-alcoholic fatty liver disease (NAFLD) group ( $p=0.046$ and 0.018 ).
\end{abstract}

(2) Iron was positively associated with body weight, BMI, waist-to-hip ratio, uric acid, liver enzymes, postprandial blood glucose, fasting insulin, HOMA-IR, triglycerides, free fatty acid, and hepatic steatosis (CAP value), and negatively associated with high-density lipoprotein cholesterol (all $p<$ $0.05)$. Iron was also positively associated with the visceral adipose area in patients with obesity and negatively associated with the subcutaneous adipose area in patients with overweight (all $p<0.05$ ). (3) Iron levels and CAP values were decreased gradually 6 months and 1 year after surgery (all $p<$ 0.05). Conclusions: Overall, our results indicated that iron is associated with hepatic steatosis in obesity. The iron level was significantly higher in patients with severe NAFLD than with mild or moderate NAFLD. LSG may reduce iron levels while improving fat deposition in the liver.

$$
\begin{aligned}
& \text { (c) } 2020 \text { The Author(s) } \\
& \text { Published by S. Karger AG, Basel }
\end{aligned}
$$

\section{Introduction}

Non-alcoholic fatty liver disease (NAFLD) is frequently associated with obesity, type 2 diabetes mellitus (T2DM), and metabolic syndrome (MS). Along with the

B.M. and H.S. contributed equally to this article. karger@karger.com www.karger.com/ofa

Karger $\stackrel{\text { ' }}{5}$

GOPEN ACCESS
(C) 2020 The Author(s)

Published by S. Karger AG, Basel

This is an Open Access article licensed under the Creative Commons Attribution-NonCommercial-4.0 International License (CC BY-NC) (http://www.karger.com/Services/OpenAccessLicense), applicable to the online version of the article only. Usage and distribution for commercial purposes requires written permission.
Xingnchun Wang or Shen Qu

Department of Endocrinology and Metabolism Shanghai Tenth People's Hospital of Tongji University 301 Yanchang Road, Shanghai 200072 (China) 2316154780@qq.com or qushencn@ hotmail.com 
increase of the above diseases, the prevalence of NAFLD is also growing [1]. NAFLD may progress to non-alcoholic steatohepatitis (NASH), or even hepatic cirrhosis and hepatocellular carcinoma [2]. However, the etiology and pathogenesis of NAFLD have not been fully elucidated.

Iron overload has been found to be associated with obesity-related diseases, such as T2DM, essential hypertension, polycystic ovary syndrome, and NAFLD [3]. Iron has been widely implicated in the pathogenesis of NAFLD and recognized as a potential target for the treatment of NAFLD. Iron induces liver oxidative stress and depletion of long-chain polyunsaturated fatty acids (LCPUFAs), n-6/n-3 LCPUFA ratio enhancement, and fat accumulation [4]. Additionally, iron may disrupt the balance between M1/M2 macrophage polarization and leads to macrophage-driven inflammation and fibrogenesis in NAFLD [5]. Therefore, there may exist an association between iron overload and hepatic steatosis.

NAFLD is closely paralleled with obesity. Laparoscopic sleeve gastrectomy (LSG) is one of the most effective methods of treating obesity. Additionally, LSG is useful as a therapeutic option to improve or reverse NAFLD. Patients with NAFLD who underwent LSG showed a significant reduction in liver steatosis and fibrosis 18 months after surgery [6]. The histology and liver function of patients with morbid obesity are significantly improved after LSG via mechanisms that involve the reduction of oxidative stress and inflammatory processes [7]. Only a few articles have focused on the change in iron levels after LSG. One previous study showed that hair loss after LSG was associated with decreased iron levels, and lower iron 1 year after LSG is more susceptible to present hair loss [8]. Therefore, study of the change in iron levels and fatty liver after LSG is warranted. Hence, this study was designed to investigate the association between iron and NAFLD in patients of Chinese ethnicity, as well as change in iron and liver steatosis after LSG.

\section{Materials and Methods}

\section{Patients}

A total of 297 subjects with obesity or overweight were enrolled from the Endocrine and Metabolism Department of Shanghai Tenth People's Hospital. Obesity was defined by a body mass index (BMI) over 28 and overweight as a BMI ranging from 25 to 28. Forty-three healthy individuals with a normal BMI (average BMI $23.22 \pm 1.32$ ) were included as controls. Among the patients with obesity, 82 subjects underwent LSG. Our inclusion criteria were: (1) age between 16 and 65 years, (2) BMI over 37.5, or BMI over 32.5 with diabetes which meets the recommended cutoff for bariatric surgery of the guidelines for surgical treatment of obesity with or without type 2 diabetes in China. The exclusion criteria were: (1) secondary cause of obesity such as hypothalamic obesity, Cushing syndrome, and hypophysis dysfunction, etc., (2) pregnancy or lactation, (3) contraindications for laparoscopic surgery, such as gastrointestinal diseases of intra-abdominal infection, adhesions, etc., (4) severe heart, liver, and kidney dysfunction, (5) organic and systemic diseases intolerant of surgery. Subjects were examined before the LSG and followed up at 6 months and 1 year post-surgery. All individual participants in this study signed informed consent forms. This study was approved by the Ethical Committee of Shanghai Tenth People's Hospital. The study conformed with the principles of the Declaration of Helsinki and the procedures followed were in accordance with institutional guidelines.

\section{Anthropometric Measurements}

Bodyweight and height were measured without shoes and with light clothing by professional staff. BMI was calculated as body weight $(\mathrm{kg}) /$ height squared $\left(\mathrm{m}^{2}\right)$. Neck circumference (NC), waist circumference (WC), and hip circumference (HC) were also measured by professional staff with a tape. The waist-to-hip ratio (WHR) was calculated by HC divided by WC. Blood pressure (BP) was also measured. Every measurement was taken twice and the average was used for the final analysis. The visceral fat area and subcutaneous fat area were measured by a fat measurement device (DHS-2000, Omron, Japan) which recorded the area of every component of the abdominal fat, and the results were expressed in square centimeters $\left(\mathrm{cm}^{2}\right)$.

\section{Laboratory Measurements}

Venous blood samples were collected from all the subjects after fasting overnight at baseline and at the 6-month and 1-year followup. Liver function including alanine transaminase (ALT), aspartate aminotransferase (AST), and gamma-glutamyl transferase $(\gamma \mathrm{GT})$, glucose metabolism including fasting blood glucose (FBG), postprandial blood glucose (PBG), fasting insulin (FINS), and 2-h post-meal insulin ( $2 \mathrm{~h}$-INS) were measured. Insulin resistance was measured by the homeostasis model assessment of insulin resistance (HOMA-IR), which was calculated by the following formula: FINS $(\mathrm{mU} / \mathrm{L}) \times$ FBG $(\mathrm{mmol} / \mathrm{L}) / 22.5$. Lipid metabolic markers (total cholesterol, triglycerides [TG], high-density lipoprotein cholesterol [HDL-C], low-density lipoprotein cholesterol [LDL-C], free fatty acid $[\mathrm{FFA}]$ ) and uric acid (UA) were also measured.

\section{Assessment of NAFLD}

Liver steatosis and fibrosis were assessed by FibroScan, a noninvasive evaluation of hepatic steatosis and fibrosis [9]. The CAP value is a marker for hepatic steatosis and the $\mathrm{E}$ value is a marker for liver fibrosis. NAFLD was diagnosed for CAP $\geq 238 \mathrm{~dB} / \mathrm{m}$ (steatosis $\geq 11 \%$ ), and according to the value of CAP the severity of NAFLD could be divided into 3 categories, including: mild NAFLD (CAP ranging from 238 to $259 \mathrm{~dB} / \mathrm{m}$, steatosis $\geq 11 \%$ ), moderate NAFLD (CAP ranging from 259 to $292 \mathrm{~dB} / \mathrm{m}$, steatosis $\geq 34 \%$ ), and severe NAFLD (CAP $\geq 292 \mathrm{~dB} / \mathrm{m}$, steatosis $\geq 67 \%)$. All subjects were evaluated by FibroScan at baseline and follow-up.

\section{Definition of $M S$}

MS was defined by 3 out of the following 4 components: (1) overweight and/or obesity (BMI $\geq 25$ ), (2) FPG $\geq 6.1 \mathrm{mmol} / \mathrm{L}$ and/or PBG $\geq 7.8 \mathrm{mmol} / \mathrm{L}$ and/or diagnosed diabetes, (3) hypertension: $\mathrm{BP} \geq 140 / 90 \mathrm{~mm} \mathrm{Hg}$ or a positive history of hypertension 
Table 1. Characteristics of the subjects

\begin{tabular}{|c|c|c|c|}
\hline & $\begin{array}{l}\text { Overweight or obesity } \\
(n=297)\end{array}$ & $\begin{array}{l}\text { Non-obesity } \\
(n=43)\end{array}$ & $p$ value \\
\hline Age, years & $33.00 \pm 10.81$ & $33.26 \pm 9.72$ & 0.882 \\
\hline Gender, male/female & $9 / 34$ & $130 / 167$ & \\
\hline Height, m & $1.69 \pm 0.08$ & $1.66 \pm 0.07$ & 0.058 \\
\hline Weight, kg & $103.55 \pm 25.51$ & $64.56 \pm 5.69$ & $<0.001$ \\
\hline BMI & $35.85 \pm 7.18$ & $23.22 \pm 1.32$ & $<0.001$ \\
\hline $\mathrm{NC}, \mathrm{cm}$ & $41.44 \pm 4.92$ & $34.79 \pm 2.52$ & $<0.001$ \\
\hline $\mathrm{WC}, \mathrm{cm}$ & $113.00 \pm 17.36$ & $82.37 \pm 5.74$ & $<0.001$ \\
\hline $\mathrm{HC}, \mathrm{cm}$ & $117.46 \pm 13.79$ & $94.44 \pm 5.24$ & $<0.001$ \\
\hline WHR & $0.96 \pm 0.07$ & $0.87 \pm 0.05$ & $<0.001$ \\
\hline $\mathrm{ALT}, \mathrm{U} / \mathrm{L}$ & $20.15(11.50-41.47)$ & $8.80(6.72-11.32)$ & $<0.001$ \\
\hline AST, U/L & $17.80(13.95-27.50)$ & $12.90(11.35-14.35)$ & $<0.001$ \\
\hline$\gamma \mathrm{GT}, \mathrm{U} / \mathrm{L}$ & $23.80(13.85-45.15)$ & $9.15(7.35-13.20)$ & $<0.001$ \\
\hline Fasting plasma glucose, $\mathrm{mmol} / \mathrm{L}$ & $5.24 \pm 1.12$ & $4.62 \pm 0.82$ & $<0.001$ \\
\hline $2 \mathrm{~h}$-postprandial plasma, $\mathrm{mmol} / \mathrm{L}$ & $7.00 \pm 3.49$ & $4.86 \pm 2.86$ & $<0.001$ \\
\hline FINS, mU/L & $17.12(8.82-29.19)$ & $5.49(4.24-7.99)$ & $<0.001$ \\
\hline $2 \mathrm{~h}-\mathrm{INS}, \mathrm{mU} / \mathrm{L}$ & $60.93(18.32-159.47)$ & $12.30(7.63-30.68)$ & $<0.001$ \\
\hline HOMA-IR & $3.83(1.88-7.09)$ & $1.11(0.81-1.56)$ & $<0.001$ \\
\hline Total cholesterol, mmol/L & $4.39 \pm 0.84$ & $4.34 \pm 0.91$ & 0.730 \\
\hline $\mathrm{TG}, \mathrm{mmol} / \mathrm{L}$ & $1.20(0.88-1.79)$ & $0.79(0.56-1.03)$ & $<0.001$ \\
\hline LDL-C, mmol/L & $2.70 \pm 0.77$ & $2.49 \pm 0.89$ & 0.102 \\
\hline $\mathrm{HDL}-\mathrm{C}, \mathrm{mmol} / \mathrm{L}$ & $1.10 \pm 0.38$ & $1.33 \pm 0.28$ & $<0.001$ \\
\hline $\mathrm{FFA}, \mathrm{mmol} / \mathrm{L}$ & $0.50(0.40-0.70)$ & $0.40(0.20-0.57)$ & 0.008 \\
\hline $\mathrm{UA}, \mathrm{umol} / \mathrm{L}$ & $407.41 \pm 103.19$ & $322.23 \pm 75.84$ & $<0.001$ \\
\hline Visceral fat, $\mathrm{cm}^{2}$ & $147.38 \pm 63.02$ & $42.60 \pm 17.98$ & $<0.001$ \\
\hline Subcutaneous fat, $\mathrm{cm}^{2}$ & $408.81 \pm 133.63$ & $140.20 \pm 59.40$ & $<0.001$ \\
\hline CAP value, $\mathrm{dB} / \mathrm{m}$ & $318.98 \pm 64.25$ & $190.70 \pm 65.26$ & $<0.001$ \\
\hline E value, $\mathrm{kPa}$ & $6.10(4.75-9.20)$ & $5.15(4.02-5.82)$ & $<0.001$ \\
\hline
\end{tabular}

Data are the mean \pm SD or median (quartile, third quartile). Statistical significance is indicated by $p<0.05$. NC, neck circumference; WC, waist circumference; HC, hip circumference; WHR, waist-to-hip ratio; ALT, alanine transaminase; AST, aspartate aminotransferase; $\gamma \mathrm{GT}, \gamma$-glutamyl transferase; FINS, fasting insulin; $2 \mathrm{~h}$-INS, 2-h post-meal insulin; HOMA-IR, homeostasis model assessment of insulin resistance; TG, riglycerides; HDL-C, high-density lipoprotein cholesterol; LDL-C, low-density lipoprotein cholesterol; FFA, free fatty acid; UA, uric acid.

or on any hypertensive treatment, (4) dyslipidemia: hypertriglyceridemia $\geq 1.7 \mathrm{mmol} / \mathrm{L}$ and/or HDL-C $<1.0 \mathrm{mmol} / \mathrm{L}$ in males or $<1.3 \mathrm{mmol} / \mathrm{L}$ in females. Additionally, hyperuricemia was defined as serum $\mathrm{UA} \geq 7 \mathrm{mg} / \mathrm{dL}(\geq 417 \mu \mathrm{mol} / \mathrm{L})$ in males, and $\geq 6 \mathrm{mg} / \mathrm{dL}$ $(\geq 357 \mu \mathrm{mol} / \mathrm{L})$ in females.

\section{Statistical Analysis}

Data were analyzed by SPSS 20.0 statistical software. Normally distributed continuous data are expressed as the mean \pm SD. Nonnormally distributed data are expressed as the median (quartile, third quartile). Categorical variables are presented as the number (percent). An independent sample $t$ test was used to compare the normally distributed data. Non-normally distributed continuous data were compared with the Mann-Whitney U test. Statistical significance before and after surgery were evaluated using the paired 2 -tailed $t$ test. The Pearson's and Spearman's correlation coefficient were used for correlations between iron levels and other markers. A $p$ value $<0.05$ was considered statistically significant.

\section{Results}

\section{Clinical Characteristics of the Subjects}

Overall, 297 overweight or obese (average age of 35.85 \pm 7.18 years and average BMI of $33.00 \pm 10.81)$ and 42 non-obese subjects (average age of $33.26 \pm 9.72$ years and average BMI of $23.22 \pm 1.32$ ) were included in the crosssectional study. Bodyweight, BMI, NC, WC, HC, WHR, $\mathrm{BP}$, liver enzymes, and UA were significantly higher in patients with overweight or obesity than the controls (all $p<0.05$ ). Disorders of glucose metabolism (FBG, PBG, INS, and HOMA-IR) and lipid metabolism (TG and FFA) were more serious in patients with overweight or obesity than the control group (all $p<0.05$ ). Additionally, as with visceral fat, subcutaneous fat was significantly higher in 
patients with overweight or obesity than in the control group (all $p<0.05$ ). When compared to the controls, patients with overweight or obesity had a higher value of $\mathrm{CAP}$ and $\mathrm{E}(318.98 \pm 64.25$ vs. $190.70 \pm 65.26 \mathrm{~dB} / \mathrm{m} ; 6.10$ [4.75-9.20] vs. 5.15 [4.02-5.82] $\mathrm{kPa}$, all $p<0.001)$. All the results are presented in Table 1 .

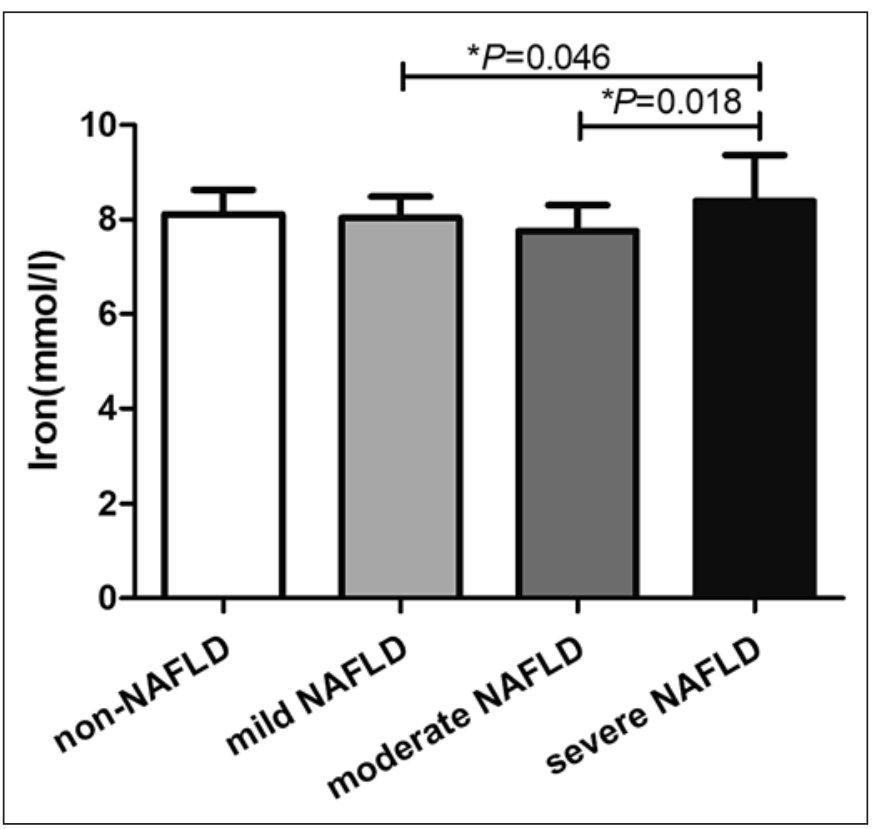

Fig. 1. Comparison of iron levels among different degrees of NAFLD.
Iron Levels in the Obesity and NAFLD Groups

Serum iron levels were significantly higher in the overweight or obesity group than in the normal BMI group $(8.18 \pm 1.47$ vs. $7.46 \pm 0.99 \mathrm{mmol} / \mathrm{L}, p=0.002)$. Besides, when the subjects were divided into groups as BMI $<25$, BMI 25-28, BMI 28-35, and BMI 35-45, we observed that the serum iron level was increased with increasing BMI (from $7.46 \pm 0.99,8.01 \pm 1.74,8.17 \pm 1.89,8.23 \pm$ 1.04 , to $8.29 \pm 0.64 \mathrm{mmol} / \mathrm{L}$, respectively, $p<0.05$ ). Serum iron levels were slightly higher in the obesity with NAFLD group than in the obesity without NAFLD group (8.30 \pm 0.92 vs. $8.30 \pm 0.92 \mathrm{mmo} / \mathrm{L}, p=0.320)$. When NAFLD was further divided into mild, moderate, and severe groups, the serum iron level was slightly higher in the severe NAFLD group than obesity without NAFLD $(8.40 \pm 0.96$ vs. $8.11 \pm 0.52 \mathrm{~mol} / \mathrm{L}, p=0.151)$, and significantly higher in the severe NAFLD group than the mild or moderate NAFLD groups $(8.40 \pm 0.96$ vs. $8.04 \pm 0.45 \mathrm{~mol} / \mathrm{L}, p=$ $0.046 ; 8.40 \pm 0.96$ vs. $7.76 \pm 0.54 \mathrm{~mol} / \mathrm{L}, p=0.018$ ), as presented in Figure 1.

\section{Comparison of Serum Iron Levels in Metabolic Disorders}

The iron level was elevated in obese patients with metabolic disorders. Serum iron was significantly higher in high $\mathrm{BP}(\mathrm{HBP})$ than in non-HBP patients $(8.44 \pm 1.50$ vs. $8.00 \pm 1.42 \mathrm{mmol} / \mathrm{L}, p=0.021)$, higher in patients with dyslipidemia than in non-dyslipidemia patients $(8.32 \pm$

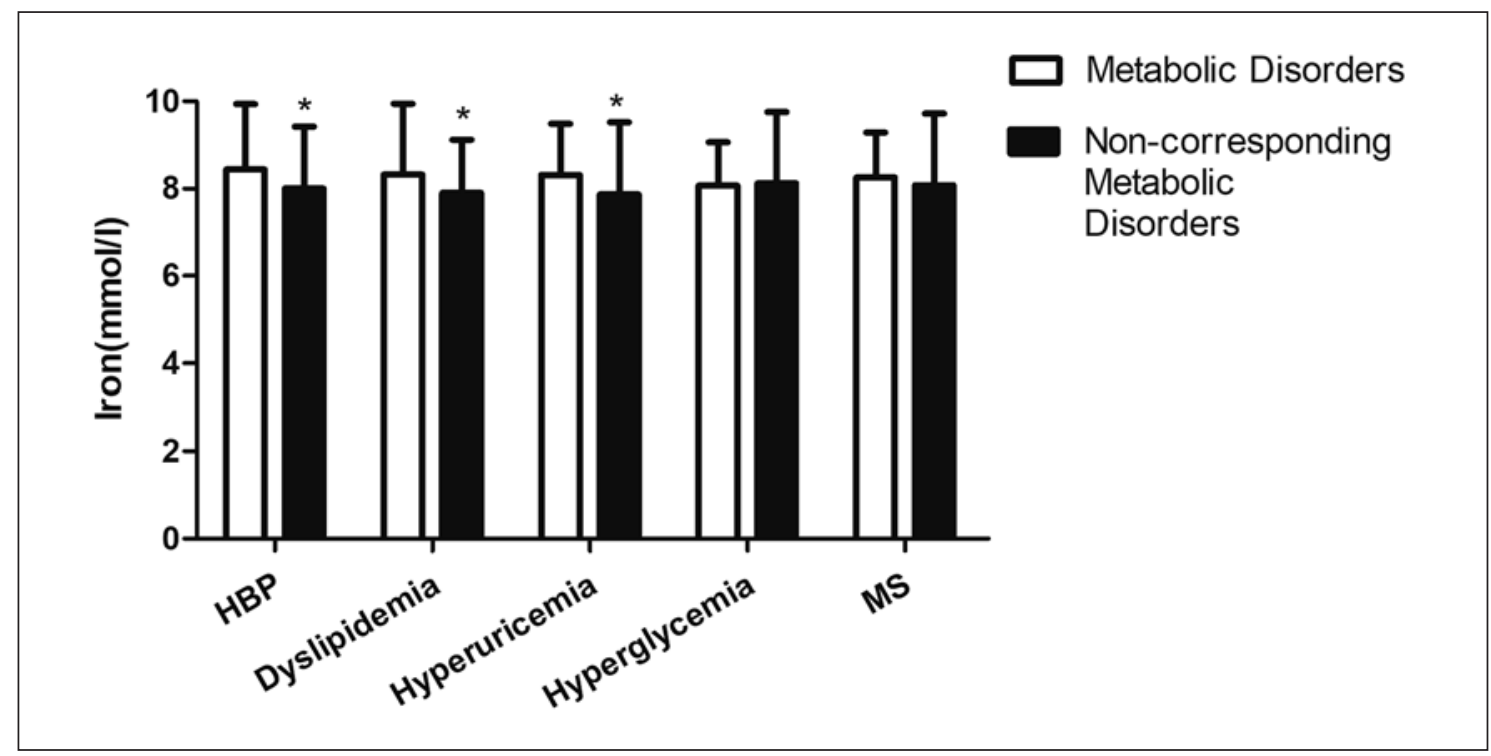

Fig. 2. Serum iron levels between metabolic disorders and non-corresponding metabolic disorders. ${ }^{*} p<0.05$, compared to non-corresponding metabolic disorders. 
Table 2. Association of iron with metabolic markers in overweight or obesity

\begin{tabular}{|c|c|c|c|}
\hline Variables & $\begin{array}{l}\text { Overweight or } \\
\text { obesity, } r(p) \\
(n=297)\end{array}$ & $\begin{array}{l}\text { Overweight, } r(p) \\
(n=36)\end{array}$ & $\begin{array}{l}\text { Obesity, } r(p) \\
(n=261)\end{array}$ \\
\hline Age, years & ns & ns & ns \\
\hline Weight & $0.300(<0.001)$ & ns & $0.304(<0.001)$ \\
\hline BMI & $0.175(0.002)$ & ns & $0.149(0.017)$ \\
\hline $\mathrm{NC}$ & $0.413(<0.001)$ & ns & $0.396(<0.001)$ \\
\hline WC & $0.239(<0.001)$ & ns & $0.021(0.002)$ \\
\hline $\mathrm{HC}$ & ns & ns & ns \\
\hline WHR & $0.229(<0.001)$ & ns & $0.221(0.001)$ \\
\hline ALT & $0.234(<0.001)$ & ns & $0.218(<0.001)$ \\
\hline AST & $0.158(0.007)$ & ns & $0.167(0.008)$ \\
\hline$\gamma \mathrm{GT}$ & $0.266(<0.001)$ & ns & $0.267(<0.001)$ \\
\hline FBG & ns & ns & ns \\
\hline PBG & $0.112(0.042)$ & ns & ns \\
\hline FINS & $0.215(<0.001)$ & ns & $0.208(0.001)$ \\
\hline 2 h-INS & $0.154(0.009)$ & ns & $0.130(0.038)$ \\
\hline HOMA-IR & $0.189(0.001)$ & ns & $0.180(0.004)$ \\
\hline Total cholesterol & ns & ns & ns \\
\hline TG & $0.186(0.001)$ & $0.375(0.019)$ & $0.151(0.015)$ \\
\hline HDL-C & $-0.179(0.002)$ & ns & $-0.168(0.007)$ \\
\hline LDL-C & ns & ns & ns \\
\hline FFA & $0.157(0.007)$ & ns & $0.135(0.031)$ \\
\hline UA & $0.224(<0.001)$ & $0.456(0.004)$ & $0.393(<0.001)$ \\
\hline Visceral fat & ns & ns & $0.323(0.029)$ \\
\hline Subcutaneous fat & ns & $-0.786(0.036)$ & ns \\
\hline CAP value & $0.183(0.021)$ & ns & $0.170(0.041)$ \\
\hline E value & ns & ns & ns \\
\hline
\end{tabular}

Statistical significance is indicated by $p<0.05$. ns, not significant; NC, neck circumference; WC, waist circumference; HC, hip circumference; WHR, waist-to-hip ratio; ALT, alanine transaminase; AST, aspartate aminotransferase; $\gamma \mathrm{GT}, \gamma$-glutamyl transferase; FBG, fasting blood glucose; PBG, postprandial blood glucose; FINS, fasting insulin; 2 hINS, 2-h post-meal insulin; HOMA-IR, homeostasis model assessment of insulin resistance; TG, triglycerides; HDL-C, high-density lipoprotein cholesterol; LDL-C, low-density lipoprotein cholesterol; FFA, free fatty acid; UA, uric acid.
1.63 vs. $7.90 \pm 1.22 \mathrm{mmol} / \mathrm{L}, p=0.008$ ), and higher in patients with hyperuricemia than in non-hyperuricemia patients $(8.31 \pm 1.18$ vs. $7.87 \pm 1.65 \mathrm{mmol} / \mathrm{L}, p=0.005)$. Moreover, the MS group manifested a higher iron level than the non-MS group $(8.25 \pm 1.04$ vs. $8.08 \pm 1.64$ $\mathrm{mmol} / \mathrm{L}, p=0.302)$, as shown in Figure 2 .

\section{Association of Iron with Metabolic Markers in \\ Patients with Overweight or Obesity}

In patients with overweight or obesity, the serum iron level was significantly positively associated with body weight, BMI, NC, WC, WHR, ALT, AST, $\gamma \mathrm{GT}$, PBG, FINS, 2 h-INS, HOMA-IR, TG, FFA, and UA, and negatively associated with HDL-C $(r=0.300, p<0.001 ; r=$ $0.175, p=0.002 ; r=0.413, p<0.001 ; r=0.239, p<0.001$; $r=0.229, p<0.001 ; r=0.234, p<0.001 ; r=0.158, p=$
$0.007 ; r=0.266, p<0.001 ; r=0.112, p=0.042 ; r=0.215$, $p<0.001 ; r=0.154, p=0.009 ; r=0.189, p=0.001 ; r=$ $0.186, p=0.001 ; r=0.157, p=0.007 ; r=0.224, p<0.001$; $r=-0.179, p=0.002)$. The above markers were significantly associated with higher iron in the obesity group (all $p<0.05$ ), while TG and UA were significantly associated with higher iron in the overweight group (all $p<$ 0.05 ). Additionally, contrary to the positive correlation between higher iron levels and visceral fat which was significant in the obesity group $(r=0.323, p=0.029)$, subcutaneous fat was negatively associated with lower iron levels in the overweight subjects $(r=-0.786, p=0.036)$. Moreover, iron was significantly positively associated with hepatic steatosis assessed by CAP value in the obesity subjects $(r=0.170, p=0.041)$. All the results are presented in Table 2. 
Table 3. Change in metabolic markers after surgical intervention (follow-up after 6 months and 1 year)

\begin{tabular}{lccc}
\hline & Pre-surgery & 6 month post-surgery & 1 year post-surgery \\
\hline BMI & $39.78 \pm 6.56$ & $29.51 \pm 4.95^{* *}$ & $28.02 \pm 5.28^{* *}$ \\
$\mathrm{NC}, \mathrm{cm}$ & $43.02 \pm 4.70$ & $37.83 \pm 3.59^{* *}$ & $36.25 \pm 3.38^{* *}$ \\
$\mathrm{WC}, \mathrm{cm}$ & $120.43 \pm 16.14$ & $99.30 \pm 13.04^{* *}$ & $93.57 \pm 15.64^{* *}$ \\
$\mathrm{ALT}, \mathrm{U} / \mathrm{L}$ & $44.25(24.90-64.92)$ & $10.90(8.80-16.60)^{* *}$ & $11.60(9.70-14.87)^{* *}$ \\
$\mathrm{AST}, \mathrm{U} / \mathrm{L}$ & $26.05(17.27-39.30)$ & $14.10(11.90-17.72)^{* *}$ & $13.70(11.97-14.87)^{* *}$ \\
$\gamma \mathrm{GT}, \mathrm{U} / \mathrm{L}$ & $39.8(24.77-61.27)$ & $13.70(9.40-19.95)^{* *}$ & $11.50(8.30-14.70)^{* *}$ \\
FBG, mmol/L & $5.87 \pm 0.91$ & $4.58 \pm 0.61^{* *}$ & $4.54 \pm 0.68^{* *}$ \\
FINS, mU/L & $24.81(18.09-40.49)$ & $8.33(5.87-12.22)^{* *}$ & $7.51(4.57-10.24)^{* *}$ \\
TG, mmol/L & $1.54(1.15-1.98)$ & $0.91(0.74-1.20)^{* *}$ & $0.80(0.67-0.98)^{* *}$ \\
HDL-C, mmol/L & $1.05 \pm 0.62$ & $1.14 \pm 0.23$ & $1.28 \pm 0.35^{* *}$ \\
FFA, mmol/L & $0.52(0.40-0.65)$ & $0.50(0.38-0.65)$ & $0.50(0.25-0.69)$ \\
UA, $\mu \mathrm{mol} / \mathrm{L}$ & $428.87 \pm 88.88$ & $372.60 \pm 94.44^{* *}$ & $355.98 \pm 89.38^{* *}$ \\
Percentage of NAFLD & $97.56(40 / 41)$ & $66.67(20 / 36)$ & $55.55(8 / 12)$ \\
\hline
\end{tabular}

Data are the mean $\pm \mathrm{SD}$, median (quartile-third quartile), or $\%(n / N) .{ }^{* *} p<0.001$. NC, neck circumference; WC, waist circumference; ALT, alanine transaminase; AST, aspartate aminotransferase; $\gamma \mathrm{GT}, \gamma$-glutamyl transferase; FBG, fasting blood glucose; FINS, fasting insulin; TG, triglycerides; HDL-C, high-density lipoprotein cholesterol; FFA, free fatty acid; UA, uric acid.

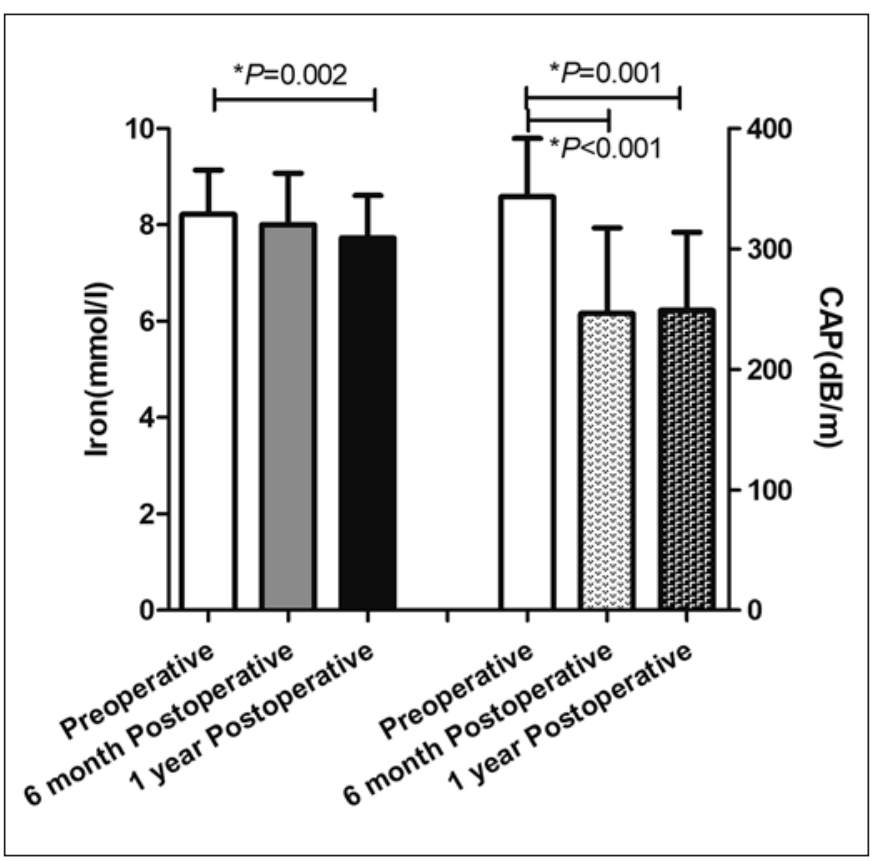

Fig. 3. Change in iron and CAP values at 6 months and 1 year postoperation.

\section{Change in Iron Levels and NAFLD after Surgery}

LSG led to decreased bodyweight, hepatic enzymes, $\mathrm{UA}$, and improved glucose-lipid metabolism at 6 months and 1 year after surgery (all $p<0.05)$, as presented in Ta- ble 3. Additionally, the iron level was decreased gradually at 6 months and 1 year after surgery (from $8.22 \pm 0.92$ to $8.00 \pm 1.07 \mathrm{mmol} / \mathrm{L}, p=0.181$, and from $8.22 \pm 0.92$ to $7.73 \pm 0.88 \mathrm{mmol} / \mathrm{L}, p=0.002)$. In parallel, the CAP value was also decreased gradually at 6 months and 1 year after surgery (from $343.42 \pm 48.33$ to $246.48 \pm 71.11 \mathrm{~dB} / \mathrm{m}, p<$ 0.001 , and from $343.42 \pm 48.33$ to $248.88 \pm 65.06 \mathrm{~dB} / \mathrm{m}$, $p=0.001$ ), as presented in Figure 3. Also, the percentage of NAFLD was decreased gradually at 6 months and 1 year after surgery (from 97.56 to $66.67 \%$ at 6 months and $55.55 \%$ at 1 year).

\section{Discussion}

Nowadays, NAFLD has been recognized as one of the major causes of chronic liver disease worldwide [10]. NAFLD is considered to be the hepatic manifestation of MS and is strongly associated with obesity, insulin resistance, and dyslipidemia [11]. NAFLD has been proven to be strongly associated with obesity, the prevalence of which increases continuously with the prevalence of overweight or obesity [1]. Insulin resistance plays a major role in the promotion of NAFLD by impairing glycogen synthesis and directing glucose into lipogenic pathways [11]. Also, the presence of dyslipidemia has been reported in $20-80 \%$ of cases associated with NAFLD [12]. Iron as a microelement plays an important role in red cell func- 
tion, oxygen transport, as well as the synthesis of protein and hormones [13]. Systemic iron overload is also related to NAFLD.

However, there are few studies on the association between iron and hepatic steatosis. Therefore, we investigated the association between iron levels and NAFLD. The results showed that serum iron levels were slightly higher in obese patients in the NAFLD group than in the obese patients without NAFLD. The serum iron level was slightly higher in the severe NAFLD group than in obese patients without NAFLD, and significantly higher in the severe NAFLD group than the mild or moderate NAFLD group. Moreover, the iron level was positively associated with visceral fat and the CAP value, which represented hepatic steatosis in obese patients.

LSG has a therapeutical effect on NAFLD. Change in body weight after LSG in obese patients with NAFLD was associated with a significant improvement in several metabolic parameters, liver enzyme levels, and liver histopathology at 18 months of follow-up [6]. Few studies have focused on iron level changes after LSG. Therefore, we investigated the change in iron level as well as hepatic steatosis after LSG. The results showed that NAFLD and hepatic steatosis percentages were decreased gradually after surgery. Furthermore, iron levels were decreased gradually at 6 months and 1 year after surgery. The improvement of NAFLD may be associated with decreased iron, and this warrants further study.

The underlying mechanism may involve lipids and insulin resistance. Dysregulation of iron metabolism results in a bidirectional relationship between the liver and visceral adipose tissue [3]. The underlying mechanism may include hepatic oxidative stress, inflammation, hepatocellular ballooning injury, lipid accumulation, or insulin resistance $[14,15]$. It was observed that iron-induced oxidative stress inhibits insulin signaling in an in vitro model of NAFLD [16]. The serum iron level was proven to be positively associated with TG and FFA, and negatively associated with HDL-C in the patients with overweight or obesity in this study. These results hint that iron may play an indirect role in NAFLD by lipid metabolism. A previous study also found that iron removal combined with a healthy diet improved both insulin sensitivity and $\beta$-cell function [3]. Therefore, iron may also play a role in glucose metabolism. In our study, we also found that iron was associated with insulin resistance valued by HOMAIR. Also, serum iron levels were significantly positively associated with PBG, FINS, and $2 \mathrm{~h}$-INS. In brief, the association of iron with both glucose and lipid metabolism may affect NAFLD in obesity.
NAFLD is a spectrum of diseases including steatosis, $\mathrm{NASH}$, cirrhosis, and end-stage liver failure [17]. The mechanism of disease progression remains unclear. $\mathrm{NASH}$ is a chronic, progressive disease characterized by fatty liver and liver cell injury with increased liver enzymes. Iron causes Fenton reactions and promotes the production of toxic reactive oxygen species $[18,19]$. The liver is susceptible to damage caused by reactive oxygen species, and iron deposition in the liver is an exacerbating factor in cases of chronic hepatitis [20]. A previous study showed that the splenic iron level was positively correlated with the severity of NASH manifestations [21]. In this study, the serum iron level was also observed to be positively associated with liver function, including ALT, AST, and $\gamma \mathrm{GT}$, which may imply that iron is also associated with NASH. Also, the liver enzymes were improved after surgery. Meanwhile, iron was decreased after surgery.

Obesity and NAFLD were closely related to MS, which is a cluster of metabolic abnormalities. There also exists an association of iron overload with MS, expressed as its components: T2DM, hypertension, polycystic ovary syndrome, and NAFLD [3]. A previous study showed that dietary iron was positively associated with the risk of MS from a sample of a total of 5,323 participants from 4 of China's megacities [22]. Another study also confirmed that total iron intake was positively associated with MS and its components in the adult population in metropolitan China based on data from 3,099 participants in the Shanghai Diet and Health Survey (SDHS) obtained during 2012-2013 [23]. In this study, serum iron levels were significantly higher in the overweight or obesity group than in the normal BMI group. Notably, the serum iron level was increased with the increase of BMI. The expression of sgk 1 induced by iron overload may be one underlying mechanism, which encodes the glucocorticoid-inducible kinase in serum and promotes the level of ferritin and fat accumulation [24]. The MS group manifested a higher iron level than the non-MS group in this study. Furthermore, serum iron was significantly higher in the HBP than the non-HBP group, higher in dyslipidemia than the non-dyslipidemia group, and higher in hyperuricemia than the non-hyperuricemia group.

\section{Conclusion}

In this study, iron levels were associated with fatty liver steatosis in obesity. The iron level was significantly higher in patients with severe NAFLD than with mild or moderate NAFLD. Additionally, LSG may reduce iron levels while improving fat deposition in the liver. 


\section{Statement of Ethics}

This study was approved by the Ethics Committee of Shanghai Tenth People's Hospital. All of the procedures performed in studies involving human participants were in accordance with the ethical standards of the national guidelines. The approval number is NCT04548232.

\section{Conflict of Interest Statement}

The authors declare that they have no conflicts of interest.

\section{Funding Sources}

This article was support by the National Natural Science Foundation of China (NSFC 81970677).

\section{Author Contributions}

B.M. performed the experiment and drafted the manuscript. H.S. helped to perform the experiment and revised the manuscript. B.Z. and S.W. participated in the data collection and statistical analysis. L.D. assisted the manuscript revision and took part in language editing. X.W. and S.Q. designed the study. All authors read and approved the final manuscript.

\section{References}

1 Younossi ZM, Koenig AB, Abdelatif D, Fazel Y, Henry L, Wymer M. Global epidemiology of nonalcoholic fatty liver disease-Meta-analytic assessment of prevalence, incidence, and outcomes. Hepatology. 2016 Jul;64(1):73-84.

2 Manne V, Handa P, Kowdley KV. Pathophysiology of Nonalcoholic Fatty Liver Disease/ Nonalcoholic Steatohepatitis. Clin Liver Dis. 2018 Feb;22(1):23-37.

3 Sachinidis A, Doumas M, Imprialos K, Stavropoulos K, Katsimardou A, Athyros VG. Dysmetabolic iron overload in metabolic syndrome. Curr Pharm Des. 2020;26(10):101924.

4 Barrera C, Valenzuela R, Rincón MA, Espinosa A, López-Arana S, González-Mañan D, et al. Iron-induced derangement in hepatic $\Delta-5$ and $\Delta-6$ desaturation capacity and fatty acid profile leading to steatosis: impact on extrahepatic tissues and prevention by antioxidant-rich extra virgin olive oil. Prostaglandins Leukot Essent Fatty Acids. 2020 Feb; 153: 102058.

5 Handa P, Thomas S, Morgan-Stevenson V, Maliken BD, Gochanour E, Boukhar S, et al. Iron alters macrophage polarization status and leads to steatohepatitis and fibrogenesis. J Leukoc Biol. 2019 May;105(5):1015-26.

6 Salman AA, Sultan AA, Abdallah A, Abdelsalam A, Mikhail HM, Tourky M, et al. Effect of weight loss induced by laparoscopic sleeve gastrectomy on liver histology and serum adipokine levels. J Gastroenterol Hepatol. 2020 Oct;35(10):1769-73.

7 Cabré N, Luciano-Mateo F, Fernández-Arroyo $S$, Baiges-Gayà $G$, Hernández-Aguilera A, Fibla M, et al. Laparoscopic sleeve gastrectomy reverses non-alcoholic fatty liver disease modulating oxidative stress and inflammation. Metabolism. 2019 Oct;99:81-9.
8 Ruiz-Tovar J, Oller I, Llavero C, Zubiaga L, Diez M, Arroyo A, et al. Hair loss in females after sleeve gastrectomy: predictive value of serum zinc and iron levels. Am Surg. 2014 May;80(5):466-71.

9 Tuong TT, Tran DK, Phu PQ, Hong TN, Dinh TC, Chu DT. Non-Alcoholic Fatty Liver Disease in Patients with Type 2 Diabetes: Evaluation of Hepatic Fibrosis and Steatosis Using Fibroscan. Diagnostics. 2020 Mar; 10(3):10.

10 Benedict M, Zhang X. Non-alcoholic fatty liver disease: an expanded review. World J Hepatol. 2017 Jun;9(16):715-32.

11 Akhtar DH, Iqbal U, Vazquez-Montesino LM, Dennis BB, Ahmed A. Pathogenesis of Insulin Resistance and Atherogenic Dyslipidemia in Nonalcoholic Fatty Liver Disease. J Clin Transl Hepatol. 2019 Dec;7(4):362-70.

12 Souza MR, Diniz MF, Medeiros-Filho JE, Araújo MS. Metabolic syndrome and risk factors for non-alcoholic fatty liver disease. Arq Gastroenterol. 2012 Jan-Mar;49(1):89-96.

13 Fernández-Real JM, Manco M. Effects of iron overload on chronic metabolic diseases. Lancet Diabetes Endocrinol. 2014 Jun;2(6):51326.

14 Choi JS, Koh IU, Lee HJ, Kim WH, Song J. Effects of excess dietary iron and fat on glucose and lipid metabolism. J Nutr Biochem. 2013 Sep;24(9):1634-44.

15 Ikura Y, Ohsawa M, Suekane T, Fukushima $\mathrm{H}$, Itabe $\mathrm{H}$, Jomura $\mathrm{H}$, et al. Localization of oxidized phosphatidylcholine in nonalcoholic fatty liver disease: impact on disease progression. Hepatology. 2006 Mar;43(3):50614.
16 Messner DJ, Rhieu BH, Kowdley KV. Iron overload causes oxidative stress and impaired insulin signaling in AML-12 hepatocytes. Dig Dis Sci. 2013 Jul;58(7):1899-908.

17 Diehl AM, Day C. Cause, Pathogenesis, and Treatment of Nonalcoholic Steatohepatitis. N Engl J Med. 2017 Nov;377(21):2063-72.

18 Crichton RR, Wilmet S, Legssyer R, Ward RJ. Molecular and cellular mechanisms of iron homeostasis and toxicity in mammalian cells. J Inorg Biochem. 2002 Jul;91(1):9-18.

19 Galaris D, Pantopoulos K. Oxidative stress and iron homeostasis: mechanistic and health aspects. Crit Rev Clin Lab Sci. 2008;45(1):123.

20 Lin TJ, Liao LY, Lin CL, Chang TA, Liu SO. Hepatic iron influences responses to combination therapy with peginterferon alfa and ribavirin in chronic hepatitis C. Hepatogastroenterology. 2008 Jul-Aug;55(85):1412-5.

21 Murotomi K, Arai S, Uchida S, Endo S, Mitsuzumi $\mathrm{H}$, Tabei $\mathrm{Y}$, et al. Involvement of splenic iron accumulation in the development of nonalcoholic steatohepatitis in Tsumura Suzuki Obese Diabetes mice. Sci Rep. 2016 Mar;6(1):22476.

22 Zhu Z, He Y, Wu F, Zhao L, Wu C, Lu Y, et al The Associations of Dietary Iron, Zinc and Magnesium with Metabolic Syndrome in China's Mega Cities. Nutrients. 2020 Feb; 12(3): 12 .

23 Zhu Z, Wu F, Lu Y, Wu C, Wang Z, Zang J, et al. Total and Nonheme Dietary Iron Intake Is Associated with Metabolic Syndrome and Its Components in Chinese Men and Women. Nutrients. 2018 Nov;10(11):10.

24 Wang H, Jiang X, Wu J, Zhang L, Huang J, Zhang Y, et al. Iron Overload Coordinately Promotes Ferritin Expression and Fat Accumulation in Caenorhabditis elegans. Genetics. 2016 May;203(1):241-53. 